\title{
Structure dependence of photochromism and thermochromism of azobenzene-functionalized polythiophenes
}

\author{
X. Y. Zhao*, M. Z. Wang \\ College of Material Science and Engineering, Hebei University of Science and Technology, 050054, Xinhua Road 505, \\ Shijiazhuang, P. R. China
}

Received 22 April 2007; accepted in revised form 28 May 2007

\begin{abstract}
Two novel azobenzene-functionalized polythiophenes, poly[4-((4-(phenyl)azo)phenoxy)butyl-3-thienylacetate] (P4) and the copolymer of 3-hexylthiophene and 4-((4-(phenyl)azo)phenoxy)butyl-3-thienylacetate (COP64) have been synthesized. The structure dependence of photoluminescence features and thermochromic behaviors of both azobenzenefunctionalized polymers was investigated. The results show that polymer structure has a strong influence on the conformation and optical properties of the resulting polythiophene derivatives. The photochemical control of photoluminescence property was achieved with homopolymer P4 using photoactive azobenzene side chains.
\end{abstract}

Keywords: smart polymers, polythiophene, photochromism, thermochromism

\section{Introduction}

The synthesis of 3 and 4-substituted polythiophenes has attracted much interest in the past decade because polythiophenes with suitable substituents not only will have better processibility and stability, but also may possess novel electrical, electrochemical and optical properties [1, 2]. Azobenzene chromophore was demonstrated to be good photo-isomerizable units [3-6] for reversible optical data storage and other electrooptic devices. Thus, the combination of polythiophene backbone with photoactive azobenzene groups could provide a new approach to develop other novel materials with unique electronic and optical properties. The polymers, for example, can exhibit a novel dual photochromism when the photoactive substituent was attached to the conjugated polythiophere backbone $[7,8]$. Similarly functionalization of the backbone of polythiophenes with azobenzene chro-

"Corresponding author, e-mail: zhaoxy66@126.com

(C) BME-PT and GTE mophore can significantly alter electrochemical and spectroelectrochemical behavior [9] as well as conductivity [10]. Therefore, the great potential for synthesis of azobenzene-functionalized polythiophene derivatives not rely solely on their ability to compete with metals or inorganic materials in existing devices but also on their use as fieldresponsive materials for completely new application.

In this paper, two azobenzene-functionalized polythiophene derivatives, poly[4-((4-(phenyl)azo)phenoxy)butyl-3-thienylacetate] (P4) and the copolymer of 3-hexylthiophene and 4-((4-(phenyl)azo) phenoxy)butyl-3-thienylacetate (COP64) were synthesized (see Figure 1), the synthesis and characterization of both azobenzene-functionalized polythiophenes were also discussed. In particular, we focus our attention on the study of structuredependent of thermochromism and photochromism, which originate from a combination of 

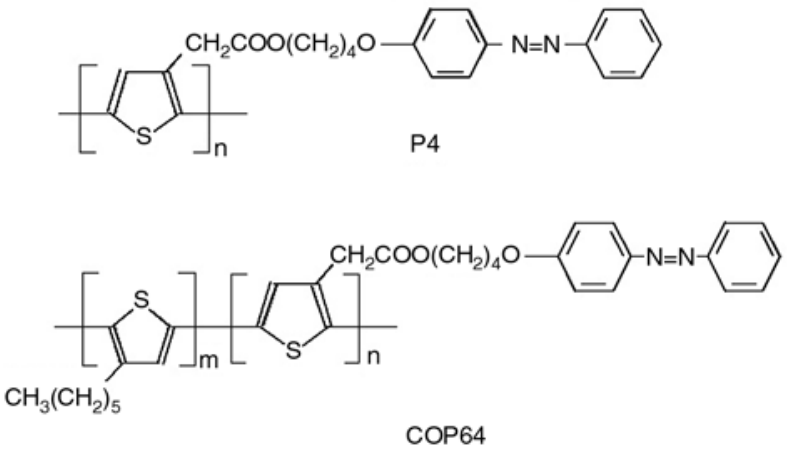

Figure 1. Chemical structure of homopolymer P4 and copolymer COP64

conjugated polythiophene backbone and photoactive azobenzene group.

\section{Experimental}

\subsection{Synthesis of 4-(4- hydroxybutyloxy)azobenzene (HBAB)}

HBAB was synthesized using a similar procedure described by Reference [11]. This reaction gave a yellow power with yield $55 \%$.

\subsection{Synthesis of 3-(4-((4-phenylazo)phe- noxyl)butyl)thienylacetate (ATh-4)}

To a two-necked flask equipped with reflux condenser, $0.97 \mathrm{~g}(6.8 \mathrm{mmol})$ of 3-thiopheneacetic acid (Aldrich) was added under $\mathrm{N}_{2}$ atmosphere, then $5 \mathrm{ml}$ dry THF and $10 \mathrm{ml}$ thionyl chloride (Aldrich) were injected into the flask with a syringe. After the solution was refluxed for $2 \mathrm{~h}$, the excess THF and unreacted $\mathrm{SOCl}_{2}$ were removed under reduced pressure at the room temperature. The solid residue was dissolved in $10 \mathrm{ml}$ dry THF, and cooled down to $0-5^{\circ} \mathrm{C}$ by ice bath, then $1.57 \mathrm{~g}$ (5.8 mmol) of 4-(4-hydroxybutyloxy)azobenzene and $1 \mathrm{ml}$ pyridine in $15 \mathrm{ml}$ of dry THF was added. After the reaction was stirred for $5 \mathrm{~h}$ and the solvent was evaporated out. The product was purified by the silica gel column chromatography using chloroform as the eluant. This reaction gave a yellow crystal (yield 74\%) with a melting point of $87.9^{\circ} \mathrm{C}$.

FT-IR $\left(\mathrm{KBr}, \mathrm{cm}^{-1}\right)$ : $3116(\mathrm{Ar} ;=\mathrm{CH}), 2864,2940$, 2963 (aliphatic; $\left.-\mathrm{CH}_{2}-\right), 1736(\mathrm{C}=\mathrm{O}), 1603,1581$, $1474(\mathrm{Ar} ; \mathrm{C}=\mathrm{C}), 1502,1441(\mathrm{~N}=\mathrm{N} ;$ cis and trans), 1248 (C-O-C; vas) 1062 (C-O-C; va), 830 (Ar; $=\mathrm{CH}, p$-substituted benzene ring), 687, 770 (Ar; $=\mathrm{CH}$, mono-substituted benzene ring). ${ }^{1} \mathrm{H}$ NMR
$\left(\mathrm{CDCl}_{3}, 400 \mathrm{MHz}, \mathrm{ppm}\right): 1.89(\mathrm{~m}, 4 \mathrm{H}), 3.69$ (s, $2 \mathrm{H}), 4.08(\mathrm{t}, 2 \mathrm{H}), 4.23(\mathrm{t}, 2 \mathrm{H}), 7.01(\mathrm{~d}, 2 \mathrm{H}), 7.08(\mathrm{~s}$, $1 \mathrm{H}), 7.3(\mathrm{~d}, 2 \mathrm{H}), 7.46(\mathrm{~s}, 1 \mathrm{H}), 7.51(\mathrm{t}, 2 \mathrm{H}), 7.92(\mathrm{~m}$, $4 \mathrm{H})$. Anal. Calcd. for $\mathrm{C}_{22} \mathrm{H}_{22} \mathrm{~N}_{2} \mathrm{SO}_{3}: \mathrm{C} 67.01, \mathrm{H}$ 5.58, N 7.11, S 8.12, O 12.18; Found C 67.03, H $5.56, \mathrm{~N} 7.10, \mathrm{~S}, 8.08$.

\subsection{Synthesis of poly[3-(4-((4-phenylazo) phenoxyl)butyl)thienylacetate] (P4)}

ATh-4 was chemically oxidized with anhydrous $\mathrm{FeCl}_{3}$ in chloroform for $5 \mathrm{~h}$. It was then washed repeatedly with water and methanol, and dedoped in aqueous ammonia. Finally the polymer was further purified in a Soxhlet apparatus with methanol for $24 \mathrm{~h}$.

\subsection{Synthesis of copolymer of 3-(4-((4- phenylazo)phenoxyl)butyl)thienylacetate and 3-hexylthiophene (COP64)}

The copolymerization was carried out by seedpolymerization method using anhydrous $\mathrm{FeCl}_{3}$ as oxidant. In a $50 \mathrm{ml}$ Schlenk reaction flask, $0.9 \mathrm{~g}$ $(5.5 \mathrm{mmol})$ of anhydrous $\mathrm{FeCl}_{3}$ was quickly put into the flask, which was subsequently evacuated and flushed with argon three times. Than $15 \mathrm{ml}$ of anhydrous $\mathrm{CHCl}_{3}$ was syringed into the reactor at $0-5^{\circ} \mathrm{C}$ followed by $0.2 \mathrm{~g}(0.51 \mathrm{mmol})$ monomer in $3 \mathrm{ml} \mathrm{CHCl}_{3}$ was added in one portion. After $10 \mathrm{~min}$, a solution of 3-hexylthiophene (3HT) in $5 \mathrm{ml}$ of $\mathrm{CHCl}_{3}$ was added in dropwise manner for $20 \mathrm{~min}$. Then the reaction mixture was stirred at the room temperature for another period of time. The polymerization reaction was terminated by the addition of $200 \mathrm{ml}$ of methanol. The precipitated polymer was then washed repeatedly with water and methanol, and dedoped in aqueous ammonia. Finally the copolymer was further purified in a Soxhlet apparatus with methanol for 24 hours.

\subsection{Characterization}

FT-IR spectra were recorded on a Perkin-Elmer System 2000 FT-IR spectrometer. NMR spectra were recorded on a BRUKER 400 NMR spectrometer at $400 \mathrm{MHz}$. Differential scanning calorimetry (DSC) analyses were performed at $5^{\circ} \mathrm{C} / \mathrm{min}$ on a TA instruments using 2920 Modulated DSC (model 2920). Molecular weights of samples were measured by gel permeation chromatography 
(GPC, Waters 410; eluent, THF) calibrated with monodispersed polystyrene standards. X-ray diffractions of polymer films on glass slides were measured using a Rigaku Rint 2000 X-ray Diffractometer. UV-visible spectra were obtained using an UV-Vis recording spectrophotometer (Shimadzu, Model UV-2501PC). For the solid-state measurements, the spectrophotometer was provided with a temperature control unit that allows measurements over a temperature range $25-250^{\circ} \mathrm{C}$ with a maximum error of $\pm 2^{\circ} \mathrm{C}$. Fluorescence measurements were conducted on a Shimadzu RF5000 spectrofluorophotometer. The photochromic experiments were carried out using an ARC lamp (Oriel, Model $68910,200-500 \mathrm{~W}$ ) as the light source. The monochromatic light peaked at 360 and $410 \mathrm{~nm}$ was isolated using filter (Model 58650 and 59285). To prepare thin films, the polymer was dissolved in chloroform, filtered, and then the solution was deposited by casting method onto clean quartz slides and finally dried under vacuum for $24 \mathrm{~h}$.

\section{Results and discussion}

All polymerization processes including homopolymerization and copolymerization in our research were carried out under lower temperature and controlled addition of monomer due to the fact that $\alpha-\alpha$ ' coupling can be considered predominant during the polymerization process carried out under these experimental conditions. The oxidative polymerization of monomer Ath-4 in the presence of $\mathrm{FeCl}_{3}$ was easy to carry out without any difficulties. For the copolymerization of ATh-4 and 3-hexylthiophene, however, in order to obtain the copolymer, the seed-oxidation polymerization technique was used. That is, the monomer ATh-4 was first initiated by $\mathrm{FeCl}_{3}$ and polymerized to form the polymer active species with moderate molecular weights, then the polymer active species was used to initiate the polymerization of 3-hexylthiophene to form copolymer. It should be also stressed that the amount of oxidant $\mathrm{FeCl}_{3}$ used in copolymerization was strictly controlled in case the formation of P3HT homopolymer in the second step.

Figure 2 illustrates the FT-IR spectra of polymer P4 and copolymer COP64, along with spectrum of the monomer ATh-4. For comparison, the spectrum of poly(3-hexylthiophene) (P3HT) is also presented in Figure 2 . The polymerization and copolymerization processes are confirmed by the disappearance of

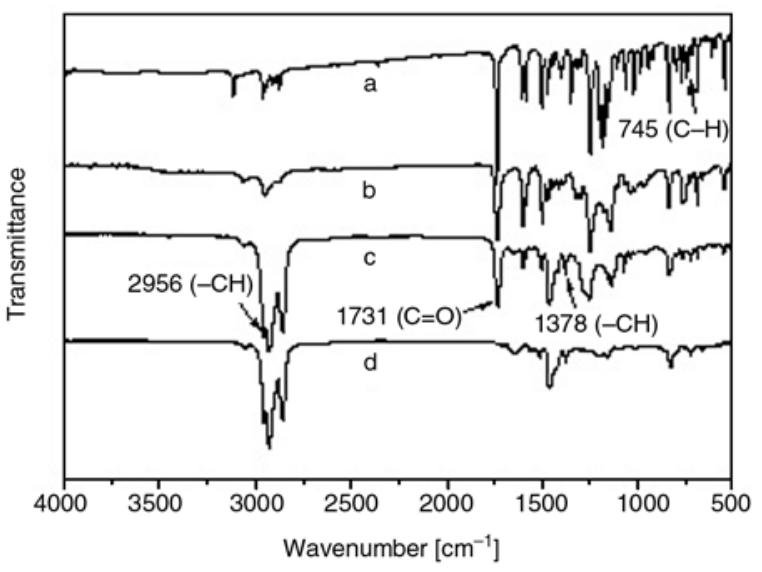

Figure 2. FTIR spectra of a) monomer ATh-4; b) P4; c) COP64 and d) P3HT

the band at $745 \mathrm{~cm}^{-1}$, which is attributable to $\mathrm{C}-\mathrm{H}_{\alpha}$ out-of-plane deformation vibration of thiophene rings. Spectral features of the ester derivatives of ATh-4 are clearly seen in the copolymer spectrum, especially the band at $1731 \mathrm{~cm}^{-1}$ and $1252 \mathrm{~cm}^{-1}$, which can be ascribable to carbonyl $(\mathrm{C}=\mathrm{O})$ stretching vibration and $\mathrm{C}-\mathrm{O}-\mathrm{C}$ stretching vibration $\left(\mathrm{v}_{a s}\right)$ respectively. Furthermore, the absorption bands occurring at $1378 \mathrm{~cm}^{-1}\left(-\mathrm{CH}_{3}, \delta_{s}\right.$ deformation vibration) and $2956 \mathrm{~cm}^{-1}\left(-\mathrm{CH}_{3}, v_{a s}\right.$ stretching vibration) in COP64 are characteristic of 3-hexylthiophene blocks, thus the present FT-IR data support the existence of Ath-4 and 3-hexylthiophene segments in copolymer. It is necessary to mention that some of FT-IR bands associated with the polythiophene backbone strongly superimpose with those originating from the chromophore. For example, the peak at $830 \mathrm{~cm}^{-1}(\mathrm{Ar} ;=\mathrm{CH}, p$-substituted benzene ring) in azobenzene is very close to the peak at $827 \mathrm{~cm}^{-1}$ associated with the out-of-plane $\mathrm{C}-\mathrm{H}_{\beta}$ deformation vibration of thiophene rings, which is characteristic of 2,3,5-trisubstituted thiophene ring. The mole ratio of 3HT unit to ATh-4 unit in COP64 determined on the basis of the NMR result is 8.62:1 $(\mathrm{mol} / \mathrm{mol})$, which is higher than that of initial feed (5.80:1). Table 1 gives physical properties of P4 and COP64.

The effects of trans-cis isomerization of photoactive chromophore on photoluminescence properties of both polymers were investigated and the corresponding results are shown in Figure 3. Upon irradiatin by the UV light of $360 \mathrm{~nm}$, the photoluminescent intensity of P4 film exhibited an obvious decrease as the UV irradiation time is raised. The real origin of this result is unclear. This is probably 
Table 1. Physical properties of P4 and COP64

\begin{tabular}{|l|c|c|c|c|c|c|c|}
\hline \multicolumn{1}{|c|}{ Samples } & Morphology & $\mathbf{M}_{\mathbf{n}}\left[\mathbf{1 0}^{\mathbf{a}}\right]$ & $\mathbf{M}_{\mathbf{w}}\left[\mathbf{1 0}^{-\mathbf{4}}\right]$ & $\mathbf{M}_{\mathbf{w}} \mathbf{M}_{\mathbf{n}}$ & $\mathbf{T}_{\mathbf{g}}\left[{ }^{\circ} \mathbf{C}\right]$ & $\mathbf{T}_{\mathbf{m}}\left[{ }^{\circ} \mathbf{C}\right]$ & $\mathbf{T}_{\mathbf{3} \%}{ }^{\mathbf{b}}\left[{ }^{\circ} \mathbf{C}\right]$ \\
\hline P4 & amorphous & 5.39 & 1.43 & 2.65 & 48.2 & - & 263.0 \\
\hline COP64 & semi-crystalline & 32.5 & 5.56 & 1.68 & 22.6 & 178.5 & 241.3 \\
\hline
\end{tabular}

aMeasured by X-ray Diffractometer; b3\% weight loss

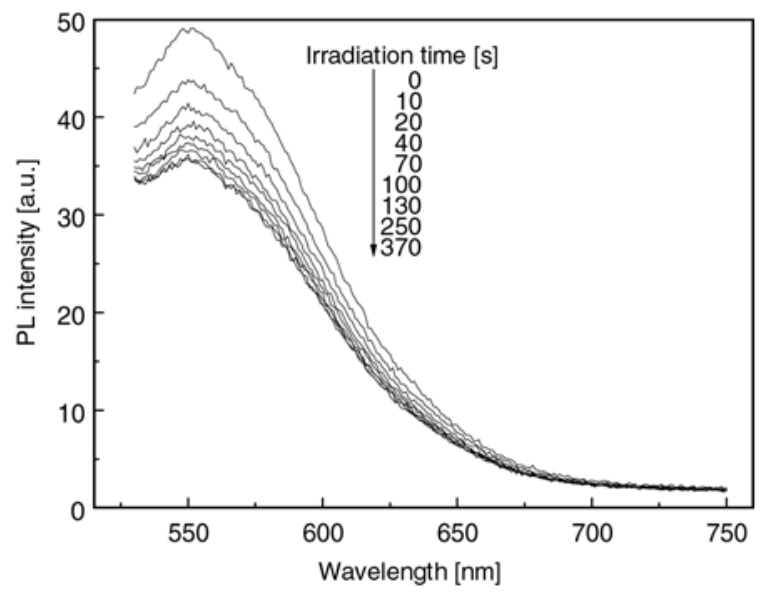

Figure 3. Photoluminescence spectra of $\mathrm{P} 4$ film for a $540 \mathrm{~nm}$ excitation wavelength with different irradiation time by the UV light at $360 \mathrm{~nm}$

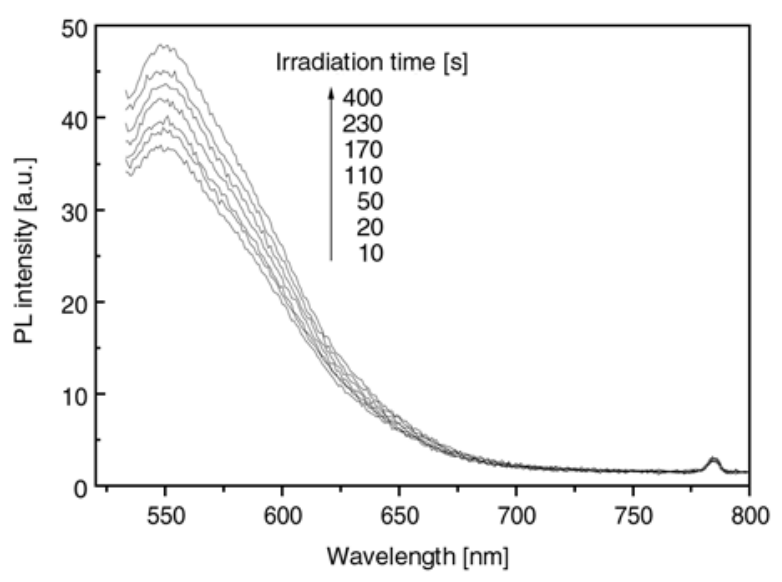

Figure 4. Photoluminescence spectra of P4 film for a $540 \mathrm{~nm}$ excitation wavelength with different irradiation time by the visible light of $410 \mathrm{~nm}$

due to the enhancement of nonradiative decay of excited species $[12,13]$ caused by the conformational transition of the conjugated polythiophene backbones with the increase of irradiation time. On the other hand, the above process is also reversible, when the irradiated samples were irradiated by the visible light of $410 \mathrm{~nm}$, the PL intensity of P4 film steadily increased up to the starting value before irradiation (see Figure 4). These results indicate that the optical properties of the functionalized polythiophene can be controlled reversibly by the photochemical isomerization of the azobenzene chromophores covalently attached to the thiophene

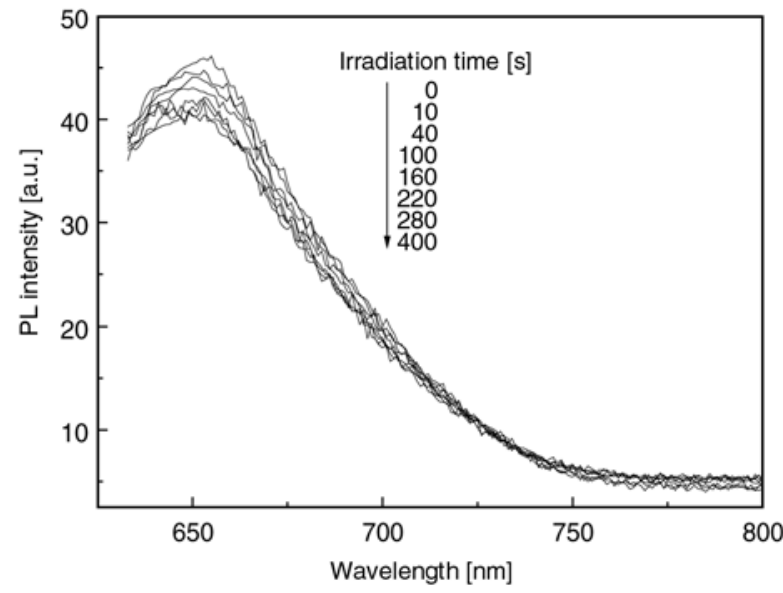

Figure 5. Photoluminescence spectra of COP64 film for a $620 \mathrm{~nm}$ excitation wavelength with different irradiation time by the UV light at $360 \mathrm{~nm}$

rings. For copolymer COP64 film, however, the photochemical isomerization of photoactive groups in the side chains showed only a quite limited effect on photoluminenscent property. (see Figure 5). When the sample COP64 film was irradiated at $360 \mathrm{~nm}$ by different UV irradiation time, only small variations in PL intensity were observed. Two explanations are possible; the higher crystallinity (due to the introduction of highly flexible hexyl groups) of COP64 as compared with P4 hinders the photochemical isomerization process, thus, lead to a little effect on PL property. Another explanation would be attributed to the lower content of photoactive azobenzene groups in COP64 in comparison with homopolymer $\mathrm{P} 4$. In other words, the trans-cis isomerization of azobenzene chromophore probably only affects the photoluminescent behavior of polythiophene conjugated units containing azobenzene side chains.

Thermochromic phenomena of neutral polythiophene derivatives may be extremely useful due to the strong blue shift in the visible region with the thermally induced disordering of the side chains [1]. The thermochromic behaviors of both polymers obtained were also investigated by heating UV-vis absorption measurements. Interestingly, no thermochromism was observed for homopolymer P4 film in addition to small variations in band absorption intensity caused by thermal cis-trans 
isomerization. This can be mainly attributed to the excess number of rigid azobenzene groups present in the side chains and also the existence of more polar carbonyl groups. It is known that the thermochromic phenomenon results from a delicate balance between repulsive side chains steric interactions and attractive interchain interactions, and the latter interactions being necessary to stabilize the low-temperature planar conformation. Since their too strong side-chain steric interactions between P4 molecules, no planar (or nearly planar) conformation is accessible for this polymer at the room temperature. In other words, at low temperature P4 will be forced to adopt a nonplanar conformation due to their strong steric hindrance. Therefore, no thermally induced planar-to-nonplanar conformational transition can take place. For COP64, however, due to the introduction of thiophene segments with highly flexible and less polar hexyl side chains, a quite obvious reversible thermochromic effect has been observed in the solid (Figure 6). Upon heating, an absorption band at $560 \mathrm{~nm}$ related to a planar (or nearly planar) conjugated backbone showed a decrease, meanwhile a new absorption band located at $446 \mathrm{~nm}$ appeared and gradually increased with increasing the heating temperature. The latter is characteristic of a delocalized twisted (less conjugated) form of the polythiophene backbone [14]. This absorbance change is fully reversible, and, upon cooling, the COP64 film recovers its initial absorption spectrum. A clear isosbestic point is observed, revealing a cooperative twisting of several repeat units upon heating and the coexistence of two conformational structures (Planar and nonplanar). The thermochromic transition behavior of COP64 is quite similar to that

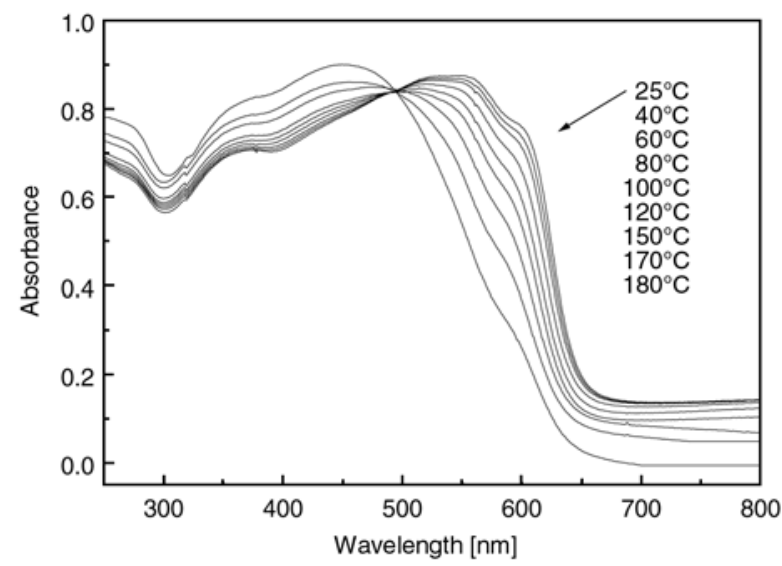

Figure 6. Temperature-dependent UV-visible absorption spectra of COP64 in the solid state

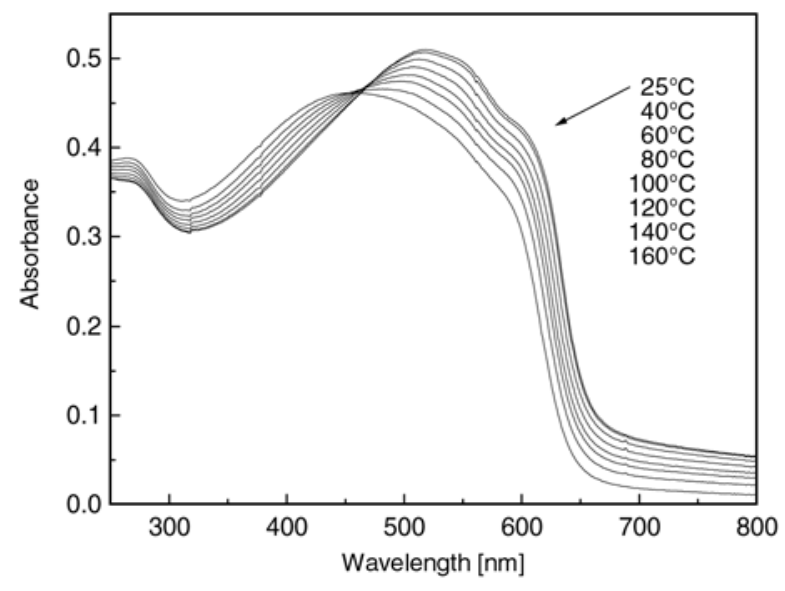

Figure 7. Temperature-dependent UV-visible absorption spectra of $\mathrm{P} 3 \mathrm{HT}$ in the solid state

of pure poly(3-hexylthiophene) (P3HT) (see Figure 7). This strong dependence of the thermochromic properties upon the type of substituents can be explained by a cooperative formation of intramolecular and intermolecular assemblies, which would be reversibly broken through thermally induced side chain disordering.

From above results, it is clear that although the optical transitions are obviously related to conformational changes of the conjugated backbone, it seems that the polymer structure such as nature of the substituents has a strong influence on the conformation and the optical properties of the resulting polythiophene derivatives.

\section{Conclusions}

In this paper, the differences in thermochromism and photochromism between two azobenzene-functionalized polythiophene derivatives have been discussed and the effect of the molecular structures of azobenzene-functionalized polymers on both chromisms was also investigated. Homopolymer P4 showed a reversible photochromic behavior induced by photochemical isomerization of side chains and no thermochromism could be observed due to strong side-chain steric interactions. As thiophene segments with highly flexible and less polar hexyl side chains were introduced into polymer chains, a quite obvious reversible thermochromic effect has been observed for COP64 in the solid. However, no significant photochromism was obtained probably due to the higher crystallinity of COP64 and also the lower content of photoactive azobenzene groups in COP64. These indicated the 
strong dependence of both chromisms upon the polymer structures.

\section{Acknowledgements}

The project was sponsored by Natural Science Foundation of Hebei Province (B2007000613). The Project was also sponsored by Department of Education of Hebei Province, and by the Scientific Research Key Foundation for Returned Overseas Chinese Scholars, Ministry of Personnel of the People's Republic of China.

\section{References}

[1] Leclerc M., Faid K.: Electrical and optical properties of processable polythiophene derivatives: structureproperty relationships. Advanced Materials, 9, 10871094 (1997).

[2] Muramatsu Y., Yamamoto T., Hasegawa M., Yagi T., Koinuma H.: Piezochromic behaviour of regioregular poly(3-hexylthiophene-2,5-diyl) and poly(5,8-dihexadecyloxyanthraquinone-1,4-diyl). Polymer, 42, 66736675 (2001).

[3] Buffeteau T., Lagygne L. F., Pezolet M., Sourisseau C.: Photoinduced orientation of azobenzene chromophores in amorphous polymers as studied by realtime visible and FTIR spectroscopies. Macromolecules, 31, 7312-7320 (1998).

[4] Zhao X. Y., Wang M. Z.: Synthesis and photoresponsive behavior of azobenzene-functionalized polythiophene films. European Polymer Journal, 42, 247-253 (2006).

[5] Berg R. H., Hvilsted S., Ramanujam P. S.: Peptide oligomers for holographic data storage. Nature, 383, 505-508 (1996).
[6] Xie S., Natansohn A., Rochon P.: Microstructure of copolymers containing disperse red 1 and methyl methacrylate. Macromolecules, 27, 1885-1890 (1994).

[7] Lévesque I., Leclerc M.: Novel dual photochomism in polythiophene derivatives. Macromolecules, 30, 4347-4352 (1997).

[8] Levesque I., Leclerc M.: Chromism in polythiophene derivatives. Synthetic Metals, 84, 203-204 (1997).

[9] Zagorska M., Kulszewicz-Bajer I., Pron A., Raimond P., Kajzar F., Attias A. J.: Polythiophenes functionalized with Disperse Red 1 chromophore. Synthetic Metals, 102, 1141-1142 (1999).

[10] Mochizuki H., Nabeshima Y., Kitsunai T., Kanazawa A., Shiono T., Ikeda T., Hiyama T., Maruyama T., Yamamoto T., Koide N.: Photochemical control of conductivity of polythiophenes with photochromic moieties. Journal of Material Chemistry, 9, 22152219 (1999).

[11] Zhao X. Y., Niu Y. H., Xiao H.: Photochemical control of photoluminescence property of photoactive polythiophenes. Journal of Polymer Science: Part B, Polymer Physics, 43, 1421-1432 (2005).

[12] Rughooputh S. D. D. V., Bloor D., Phillips D., Movaghar B.: One-dimensional exciton diffusion in a conjugated polymer. Physical Review B, 35, 8103 8112 (1987).

[13] Linton J. R., Frank C. W., Rughooputh S. D. D. V.: Fluorescence studies of poly(3-hexylthiophene) solutions. Synthetic Metals 28, 393-398 (1989).

[14] Lévesque I., Leclerc M.: Ionochromic and thermochromic phenomena in a regioregular polythiophene derivative bearing oligo(oxyethylene) side chains. Chemistry of Materials, 8, 2843-2849 (1996). 